\title{
Small airway dysfunction in well-treated never-smoking HIV-infected individuals
}

\author{
To the Editor:
}

Global projections from the World Health Organization rank chronic obstructive pulmonary disease (COPD) and HIV as the third and eighth leading causes of death by 2030, respectively [1]. An increasingly large number of individuals will consequently face a double burden of disease. The incidence of COPD is relatively high in the HIV-infected population [2], and HIV has been shown to be an independent risk factor $[3,4]$.

Previous studies might be susceptible to confounding factors, as they have included current or previous smokers, patients who had not been receiving combination antiretroviral therapy (cART), patients co-infected with hepatitis $\mathrm{C}$ virus (HCV), and cocaine or marijuana users. It is therefore unclear whether HIV infection per se is associated with airway abnormalities, which could lead to increased susceptibility to noxious stimuli, such as smoke or biomass fuel.

The initial locus of inflammation in many lung diseases is confined to small conducting airways, a development that could precede emphysematous destruction [5]. Significant small airway disease must develop before the forced expiratory volume in $1 \mathrm{~s}$ (FEV1) becomes abnormal. The lung clearance index (LCI) can be used to study airway disease during the "silent years", which is the time between the onset of pathology and detection of disease, using conventional lung function tests $[6,7]$. We hypothesised that well-treated HIV, in the absence of non-HIV risk factors, is associated with an increased LCI.

For complete methodology and data, please refer to the Dryad data repository (https://doi.org/10.5061/ dryad.486t8). HIV-infected individuals, aged 30-70 years from the ongoing Copenhagen Co-morbidity in HIV infection (COCOMO) study who were receiving cART at the outpatient clinic at Rigshospitalet, Copenhagen, Denmark (NCT02382822) were included, and the study was approved by the Regional Ethics Committee of Copenhagen (H-15017350) and the Danish Data Protection Agency (RH-2016-20: 04369). Written informed consent was obtained from all participants. Eligibility criteria for all participants included: no current or previous history of smoking or drug use, absence of HCV co-infection, no self-reported respiratory disease, nor use of any type of inhalation medication. Control subjects were found through advertisement at the outpatient clinic, among hospital staff, and their relatives. The St. George's Respiratory Questionnaire for COPD patients (SGRQ-C) was evaluated using suitable software [8], and inflammatory markers (high sensitivity C-reactive protein, white blood cells, fibrinogen and CD4/CD8 ratio) were assessed. Spirometry predicted values and $z$-scores were calculated using Global Lung Function Initiative equations [9]. The spirometric lower limit of normal (LLN) was defined as the fifth centile of the reference population (equivalent to $-1.64 z$-scores). The Exhalyzer D device (Eco Medics AG, Duernten, Switzerland) was used according to the American Thoracic Society/European Respiratory Society consensus statement on multiple breath washout (MBW) tests [10]. Inhomogeneity arising at the conducting and acinar airway zones, represented by Scond and Sacin, respectively, was derived from the $\mathrm{MBWN}_{2}$ lung clearance index (LCI). We used reference equations provided by HoulTz et al. [11] that were obtained using the same equipment, as well as the most recently updated reference equations by VERBANCK et al. [12] that were obtained using different equipment. The upper limit of normal (ULN) for the LCI was strictly defined as $1.96 \mathrm{z}$-scores to limit false positive values.

The primary objective was to determine the differences in LCI between HIV-infected individuals and controls. To find a clinically relevant difference $(\Delta)$ of 1 unit in the LCI, in a two sample t-test with $S D=1$, $\alpha=0.05$, and power $=0.85$, would require $n=18$ in a balanced design. Clinical factors associated with the LCI

@ERSpublications

Well preserved spirometry but evidence of small-airway dysfunction in never-smoking HIV-infected individuals http://ow.ly/oZCC308iUpo

Cite this article as: Ronit A, Mathiesen IH, Gelpi M, et al. Small airway dysfunction in well-treated neversmoking HIV-infected individuals. Eur Respir J 2017; 49: 1602186 [https://doi.org/10.1183/ 13993003.02186-2016]. 
were assessed in a linear regression model. Variables were included in multiple regression if they had a univariate association with LCI at $\mathrm{p}<0.05$. Explanatory variables were chosen a priori and included age, body mass index, gender and HIV status. Statistical analyses were performed using the R-Project (V.3.3.0).

65 individuals were recruited, including $45 \mathrm{HIV}$-infected individuals and 20 controls. Most were able to perform reliable $\mathrm{MBWN}_{2}$ measurements; however, seven tests were discarded because of $\mathrm{N}_{2}$-leakage or cough. These individuals were excluded from the primary analysis. The mean $\pm \mathrm{sD}$ age $(48.9 \pm 10.0)$ versus $(46.3 \pm 13.4)(p=0.41)$, gender $(\mathrm{p}=0.42)$, ethnicity $(\mathrm{p}=0.76)$, and all other demographic variables, as well as exposure to second-hand smoke, were comparable between the two groups (see Dryad data repository for a full characterisation). All individuals had never smoked, nor used marijuana, crack cocaine or intravenous drugs, and all were HCV antibody negative. One HIV-infected individual and one uninfected control reported the use of asthma medication during childhood. The median (interquartile range) time from confirmation of HIV diagnosis was 11.9 (6.4-18) years. All had a fully suppressed viral load. The mean \pm SD CD4 was $655 \pm 258$ and CD4 nadir was $292 \pm 187$. None of the participants had a history of Pneumocystis pneumonia, and only one individual had a previous history of AIDS (Kaposi's sarcoma). HIV-infected individuals presented with more subjective respiratory morbidity (SGRQ-C total: 3.5 (1.8$6.3)$ versus $1.4(0.7-3.4)(\mathrm{p}<0.01)$; activity: $7.6(0-14.8)$ versus $0(0-7.6)(\mathrm{p}=0.02)$; impact: $0(0-0)$ versus 0 $(0-0)(\mathrm{p}=0.02)$; symptoms: $10.3(0-19.2)$ versus $5.6(0-10.3)(\mathrm{p}=0.14)$.

Dynamic lung volumes were normal to slightly reduced in HIV-infected individuals and controls (figure 1). FEV1/FVC $z$-scores $(\mathrm{p}=0.21)$ were similar between the two groups, and only one HIV-infected individual and two controls met the LLN criteria. Among the HIV-infected individuals, 41\% (95\% CI $27-56 \%$ ) met the ULN criteria for the LCI, whereas 5\% (95\% CI 1-24\%) of the controls had an abnormal LCI $(\mathrm{p}<0.01)$ (figure 1$)$. When different reference equations were used, these values were $18 \%$ and $0 \%$, respectively [12].

The LCI and FEV1 z-scores were not correlated (figure 1). Similarly, the other $\mathrm{MBWN}_{2}$ indices (i.e. Scond and Sacin) were not correlated with FEV1 (data not shown). Following univariate analysis, the only clinical variables associated with LCI were age $(\beta=0.23$ per decade (95\% CI $0.02-0.44) ; p=0.03$ ) and HIV status ( $\beta=0.62$ (95\% CI $0.15-1.08) ; p=0.01$ ). In multivariable analysis, only HIV status was significantly associated with the LCI ( $\beta=0.55$ (95\% CI 0.09-1.01), $\mathrm{p}=0.019)$. The zenith viral load, CD4, and CD4 nadir were not associated with LCI, and fibrinogen was the only biomarker that was significantly associated with LCI $\left(\mathrm{R}^{2}=0.17, \mathrm{p}=0.01\right)$. Neither the LCI $\left(\mathrm{R}^{2}=0.01, \mathrm{p}=0.44\right)$ nor the FEV1 $z$-score $\left(\mathrm{R}^{2}=0.01, \mathrm{p}=0.49\right)$ were correlated with the log-transformed global SGRQ score.
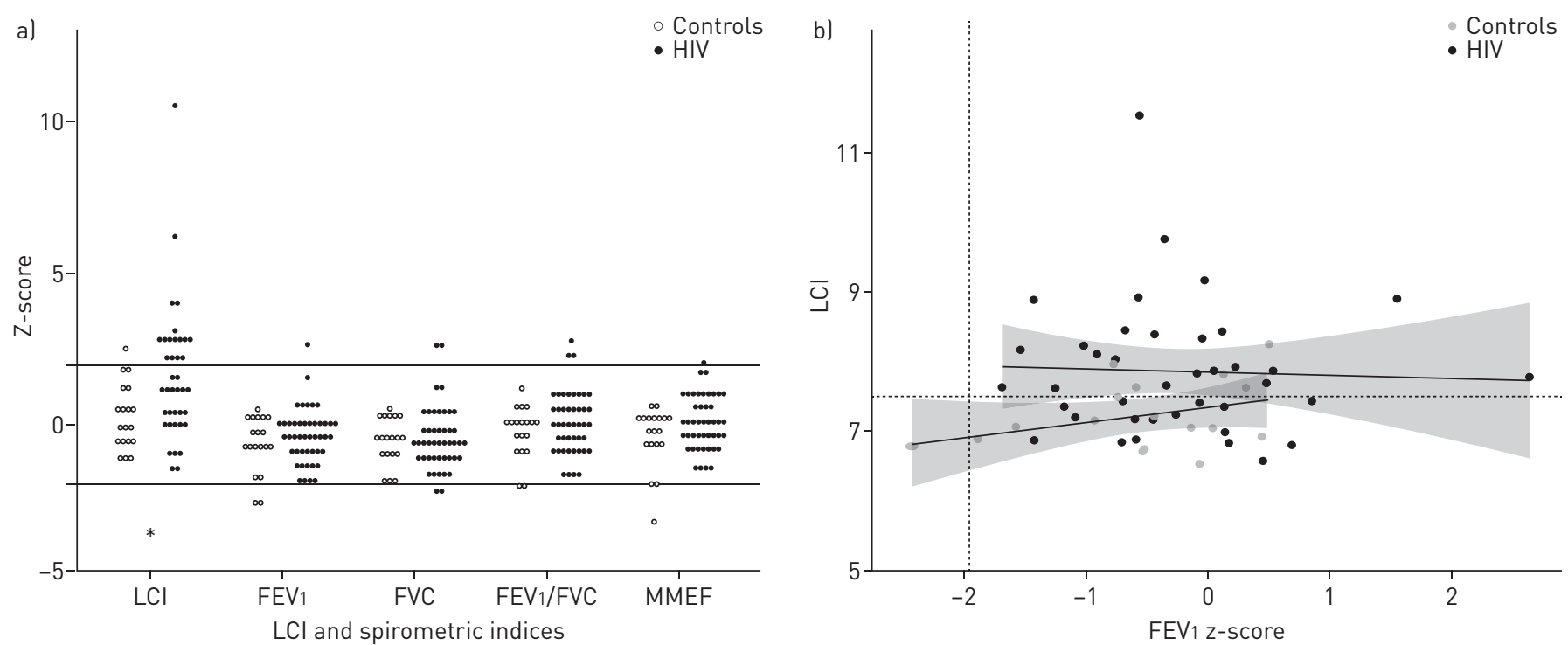

FIGURE 1 a) Dot plots depicting pulmonary function parameters in HIV-infected individuals and healthy controls. Horizontal lines indicate \pm 1.96 $z$-scores. *: $\mathrm{p}<0.01$ for the difference of absolute mean compared with uninfected controls. b) Scatterplots with regression lines for LCl predicted by forced expiratory volume in $1 \mathrm{~s}\left(F_{E V}\right) z$-score for HIV-infected individuals and uninfected controls. Coefficient of determination and significance values were $R^{2}=0.002, p=0.80$ and $R^{2}=0.14, p=0.13$ for HIV-infected individuals and uninfected controls, respectively. Omitting the two data points with the highest FEV1 z-scores did not change the regression line $\left(R^{2}=0.018, p=0.4\right)$. FVC: forced vital capacity; $L C l$ : lung clearance index; $M M E F$ : maximum mid-expiratory flow. 
Taken collectively, this is the first study to assess $\mathrm{MBWN}_{2}$ in $\mathrm{HIV}$-infected individuals. Our findings suggest that HIV might be associated with small airway dysfunction in well-treated individuals, with normal spirometry values, and an absence of confounding factors. Previous studies have primarily used conventional pulmonary function analysis in this population. Although the Strategic Timing of AntiRetroviral Treatment (START) trial lacked information on respiratory risk factors, almost half of the participants with COPD reported lifelong non-smoking [13]. Other studies reported a reduced diffusing capacity for carbon monoxide (DLCO) in HIV-infected smokers and non-smokers, with a striking 19 out of 40 HIV never-smokers exhibiting a DLCO $<80 \%$ predicted [14], and eight out of 30 exhibiting a DLCO $\leqslant 60 \%$ predicted [15]. These findings suggest that HIV is a causative factor. Nevertheless, these studies were vulnerable to various confounders, e.g. the absence of cART, HCV co-infection, and marijuana and cocaine use $[14,15]$. Our study gives further support to the hypothesis that HIV might be independently associated with airway abnormalities. Underlying mechanisms have not been elucidated, but might include inflammation, protease-antiprotease imbalance, oxidative stress, and altered lung microbiome, among others.

Our study has limitations. We were unable to determine the location of ventilation inhomogeneity, and Scond and Sacin were not significantly increased in HIV-infected individuals. Our study might also be susceptible to unmeasured residual confounding, such as passive smoking, and various early life events, which are difficult to quantify.

In summary, despite well-preserved spirometry, HIV was found to be associated with a higher LCI. Serial measurements with comparison of $\mathrm{MBWN}_{2}$ results with pathophysiological, microbiological, and radiological measures could facilitate a better understanding of the natural history of HIV-associated lung disease.

Andreas Ronit ${ }^{1}$, Inger Hee Mathiesen ${ }^{2}$, Marco Gelpi ${ }^{1}$, Thomas Benfield ${ }^{3}$, Jan Gerstoft ${ }^{1}$, Tanja Pressler ${ }^{2}$, Anders Christiansen ${ }^{4}$, Jens Lundgren ${ }^{5}$, Jørgen Vestbo ${ }^{6}$ and Susanne Dam Nielsen ${ }^{1}$

${ }^{1}$ Viro-immunology Research Unit, Dept of Infectious Diseases 8632, Rigshospitalet, University of Copenhagen, Copenhagen, Denmark. ${ }^{2}$ Copenhagen Cystic Fibrosis Center, Dept of Infectious Diseases, Rigshospitalet, University of Copenhagen, Copenhagen, Denmark. ${ }^{3}$ Dept of Infectious Diseases, Hvidovre Hospital, University of Copenhagen, Denmark. ${ }^{4}$ Dept of Respiratory Medicine, Faculty of Health Sciences, Southern University of Denmark, Odense, Denmark. ${ }^{5} \mathrm{CHIP}$, Dept of Infectious Diseases, Rigshospitalet, University of Copenhagen, Copenhagen, Denmark. ${ }^{6}$ Division of Infection, Immunity and Respiratory Medicine, University of Manchester, Manchester, UK.

Correspondence: Andreas Ronit, Viro-immunology Research Unit, Dept of Infectious Diseases 8632, Copenhagen University Hospital, Blegdamsvej 9B; DK-2100 Copenhagen Ø; Denmark. E-mail: andreas.ronit@regionh.dk

Received: Nov 072016 | Accepted after revision: Nov 302016

Support statement: This work was supported by Rigshospitalet Research Council and the Novo Nordisk Foundation. The study was designed, conducted, analysed and written by the authors without the involvement of any commercial party. Funding information for this article has been deposited with the Crossref Funder Registry.

Conflict of interest: Disclosures can be found alongside this article at erj.ersjournals.com

Acknowledgements: We thank all the study subjects for their participation.

Contributors: A. Ronit and I.H. Mathiesen designed the study. A. Ronit, I.H. Mathiesen, M. Gelpi, and S.D. Nielsen collected the data. A. Ronit was responsible for the statistical analysis. All authors interpreted the data. A. Ronit drafted the manuscript. All authors have critically revised and approved the final version. A. Ronit is the guarantor of the study.

\section{References}

1 World Health Organization. Global Health Estimates Summary Tables: Projection Of Deaths By Cause, Age And Sex, By World Bank Income Group And WHO Region. Geneva, World Health Oraganization, 2013. Available from: http://www.who.int/healthinfo/global_burden_disease/GHE_DthWHOReg6_Proj_2015_2030.xls?ua=1

2 Makinson A, Hayot M, Eymard-Duvernay S, et al. High prevalence of undiagnosed COPD in a cohort of HIV-infected smokers. Eur Respir J 2015; 45: 828-831.

3 Crothers K, Huang L, Goulet JL, et al. HIV infection and risk for incident pulmonary diseases in the combination antiretroviral therapy era. Am J Respir Crit Care Med 2011; 183: 388-395.

4 Crothers K, Butt AA, Gibert CL, et al. Increased COPD among HIV-positive compared to HIV-negative veterans. Chest 2006; 130: 1326-1333.

5 McDonough JE, Yuan R, Suzuki M, et al. Small-airway obstruction and emphysema in chronic obstructive pulmonary disease. N Engl J Med 2011; 365: 1567-1575.

6 Horsley A. Lung clearance index in the assessment of airways disease. Respir Med 2009; 103: 793-799.

7 Rowan SA, Bradley JM, Bradbury I, et al. Lung clearance index is a repeatable and sensitive indicator of radiological changes in bronchiectasis. Am J Respir Crit Care Med 2014; 189: 586-592.

8 Gelpi M, Argentiero J, Jones PW, et al. A Scoring Application for the St. George's Respiratory Questionnaire. Chest 2016; 150: 747-748.

9 Quanjer PH, Stanojevic S, Cole TJ, et al. Multi-ethnic reference values for spirometry for the 3-95-yr age range: the global lung function 2012 equations. Eur Respir J 2012; 40: 1324-1343.

10 Robinson PD, Latzin P, Verbanck S, et al. Consensus statement for inert gas washout measurement using multiple- and single-breath tests. Eur Respir J 2013; 41: 507-522. 
11 Houltz B, Green K, Lindblad A, et al. Tidal $\mathrm{N}_{2}$ washout ventilation inhomogeneity indices in a reference population aged 7-70 years. Eur Respir J 2012; 40: 3797.

12 Verbanck S, Van MA, Schuermans D, et al. Transfer factor, lung volumes, resistance and ventilation distribution in healthy adults. Eur Respir J 2016; 47: 166-176.

13 Kunisaki KM, Niewoehner DE, Collins G, et al. Pulmonary function in an international sample of HIV-positive, treatment-naive adults with CD4 counts $>500$ cells $/ \mu \mathrm{L}$ : a substudy of the INSIGHT Strategic Timing of AntiRetroviral Treatment (START) trial. HIV Med 2015; 16: Suppl. 1, 119-128.

14 Gingo MR, George MP, Kessinger CJ, et al. Pulmonary function abnormalities in HIV-infected patients during the current antiretroviral therapy era. Am J Respir Crit Care Med 2010; 182: 790-796.

15 Gingo MR, He J, Wittman C, et al. Contributors to diffusion impairment in HIV-infected persons. Eur Respir J 2014; 43: 195-203. 